\title{
Predication and Analysis of Epileptic Seizure Neurological Disorder using Intracranial Electroencephalography (iEEG)
}

\author{
SANJAY S. PAWAR, *SANGeETA R. ChOUgUle, \\ Ph.D. Scholar, Shivaji University Kolhapur, Assistant Professor, Bharati Vidyapeeth's College of \\ Engineering, Kolhapur, (Maharashtra), INDIA. \\ sanjaypawar832@gmail.com \\ *Professor, Kolhapur Institute of Technology College of Engineering, Kolhapur, (Maharashtra), \\ INDIA. \\ shivsangeeta.chougule@rediffmail.com
}

\begin{abstract}
Epileptic seizure is one of the neurological brain disorder approximately 50 million of world's population is affected. Diagnosis of seizure is done using medical test Electroencephalography. Electroencephalography is a technique to record brain signal by placing electrodes on scalp. Electroencephalography suffers from disadvantage such as low spatial resolution and presence of artifact. Intracranial Electroencephalography is used to record brain electrical activity by mounting strip, grid and depth electrodes on surface of brain by surgery. Online standard Intracranial Electroencephalography data is analyzed by our system for predication and analysis of Epileptic seizure. The pre-processing of Intracranial Electroencephalography signal is done and is further analyzed in wavelet domain by implementation of Daubechies Discrete Wavelet Transform. Features were extracted to classify as preictal and ictal state. Analysis of preictal state was carried out for predication of seizure. Intracranial Electroencephalography signals provide better result and accuracy in seizure detection and predication. Earlier warning can also be issued to control seizure with anti- epileptic drugs.
\end{abstract}

Keywords-Artifact, Daubechies Discrete Wavelet transform, Epileptic Seizure, Intracranial Electroencephalography, Seizure Classification, Seizure Predication.

Received: August 1, 2020. Revised: December 13, 2020. Accepted: December 27, 2020. Published: December $31,2020$.

\section{Introduction}

The status of human whole body is represented by brain function. Central Nervous System (CNS) consists of neurons which transmits information in responses to simulation. Simulation of neuron generates action potential in the voltage range between negative $60 \mathrm{mv}$ to positive $10 \mathrm{mv}$ and the action potential generated remains for 5 Mill second to 10 Mill seconds. Scalp Electroencephalography is used for recording of brain signal to diagnosis many neurological disorders and to detect abnormalities in human body. Electroencephalography signals are used for investigation of Epileptic seizure and testing of drug effect on Epilepsy patients [1]. Scalp Electroencephalography suffers from disadvantage such as low spatial resolution and the signals acquired may be contaminated with noises which are called as artifacts. Presence of artifact affects the performance of seizure detection system and predication of seizure is also difficult in scalp Electroencephalography. Intracranial Electroencephalography (iEEG) is a clinical technique where strip, grid or depth electrodes are implanted on surface of brain by surgery to monitor brain activities. Intracranial Electroencephalography helps in exact diagnosis of epilepsy syndrome and in planning of drug management for epileptic surgery. Intracranial Electroencephalography confirm region of seizure for planning epilepsy surgery. Development of seizure predication system may enhance quality, safety and life of patient.

Detection of Epileptic seizures using Electroencephalography (EEG) dataset and Intracranial Electroencephalography (iEEG) dataset has attracted many researchers to develop various algorithms with good sensitivity, specificity, accuracy and True predicative value. Automatic seizure detection was carried out using wavelet decomposition in five scales of multi-channel intracranial EEG. Features such as Energy, relative amplitude, coefficient of variation and fluctuation index were extracted and classified using support vector machine for seizure detection [2]. Oneclass support vector machine novelty detection method was implemented for detecting of seizure using Intracranial Electroencephalography. Short-time, energy-based statistics were computed. Validation of detector was done using leave- 
one-out cross-validation [3]. Seizure detection was carried out using Lacunarity and Bayesian Linear Discriminant Analysis (BLDA) using long-term Freiburg intracranial EEG dataset. Wavelet decomposition was done up to five scale and features such as Lacunarity and Fluctuation index were extracted. The algorithm gives a sensitivity of $96.25 \%$. [4].

Accurate Seizure detection or Ictal stage detection and predication of pre-ictal stage still remains to be challenging area for researchers. The objective of research is to develop accurate seizure detection tool to find Ictal stage and predication of pre-ictal stage to predicate seizure. Development of predication algorithm will issue earlier warning of seizure to patient and with intake of anti- epileptic drug further seizure can be prevented. The development of algorithm will help to improve the quality of life of Epileptic patient.

\section{Intracranial Electroencephalography}

Intracranial Electroencephalography is a recording method of brain signals by implanting the electrodes. The electrodes are of grid, strip and depth electrodes placed over the surface of brain. For acquiring precision localization of seizure depth and grid electrodes are implanted in specific region on surface of brain. The brain signal amplitude recorded by such type of electrodes is of higher magnitude as compare to the signal recorded by scalp EEG. The brain signals received from Intracranial Electroencephalography (iEEG) are free from muscle, eye blink and many other artifacts which are present in scalp EEG. The invasive electrodes recording can only be used for Epileptic seizure suffering patient. The implantation of Intracranial Electroencephalography helps in exact diagnosis of epileptic seizure and in planning of drug management for prevention of epileptic seizure. Intracranial Electroencephalography is also useful for planning epilepsy surgery. The region of brain surgery can confirmed after various testing of Intracranial Electroencephalography. The advantage of Intracranial Electroencephalography (iEEG) is its flexibility in electrode placement and recording. Intracranial Electroencephalography achieves greater sensitivity, precision and spatial resolution as compare to scalp EEG. Intracranial Electroencephalography is also known as Electrocorticography $(\mathrm{ECoG})$ is shown in Figure 1.

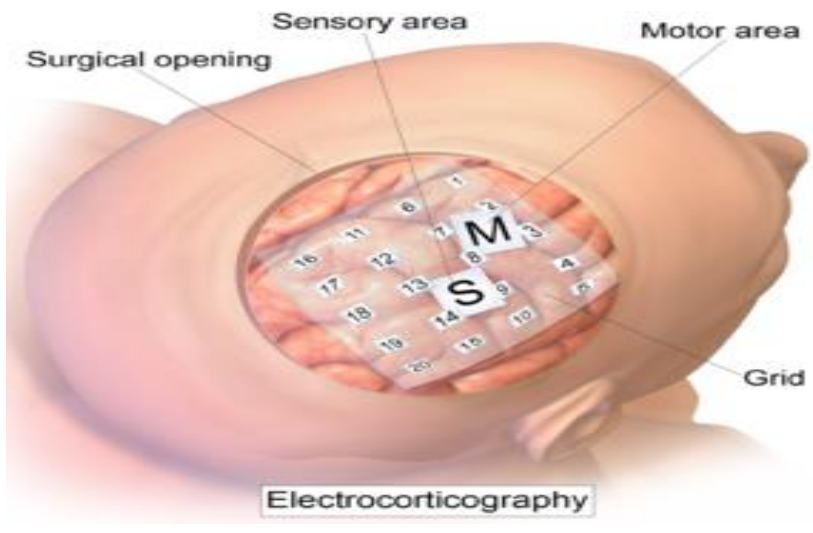

Figure 1: Electrocorticography (ECoG)

The electrodes are mounted on surface of brain to record electrical activity from the cerebral cortex. The surgery needs to be carried out to access the cortex. The surgeon must first remove part of skull to access the brain surface. The electrodes are then implanted on cortex surface as per placement guided by EEG. The grids used are flexible and transparent and the spacing between two electrodes is kept $1 \mathrm{~cm}$ apart and has a diameter of $5 \mathrm{~mm}$. To measure the deeper activity in the brain the depth electrodes are used. Intracranial Electroencephalography also suffers certain disadvantages such as limited area of exposed cortex.

The signals generated from brain are called as brain rhythms. The brain rhythm changes its amplitude and frequency in presence of abnormality. There are five major brain waves categories with respect to their frequencies namely named as alpha, beta, gamma, delta and theta. The delta waves are from 0.5 to $4 \mathrm{~Hz}$ and are mainly associated with deep sleep. The theta waves are from 4 to $7.5 \mathrm{~Hz}$ and are associated with drowsiness and meditation. The alpha waves are from 8 to $13 \mathrm{~Hz}$ and are associated with awareness without concentration and they appeared to be rounding sinusoidal in shape. The beta waves are from $14 \mathrm{~Hz}$ to $30 \mathrm{~Hz}$ and are associated with walking, thinking and problem solving. The frequencies above $30 \mathrm{~Hz}$ up to $60 \mathrm{~Hz}$ are called as gamma waves which has low amplitude and are rarely used investigation and analysis process [1],[5].

The brain wave patterns are used in identification and classification of many neurological diseases out of which one is Epileptic seizures. Epileptic seizure is a neurological disorder which arises due to excessive electrical discharge from brain cells. There is change in amplitude and frequency during onset of seizure. The Electroencephalogram brain signal measurement technique remains to be very effective method to diagnosis and analyze the epileptic seizure in time domain and wavelet domain using various higher order feature vectors and classification algorithm [6][7][8]. 


\section{Seizure Predication and Classification System}

Seizure classification is a binary classification problem in which one class is of ictal stage and the other class belongs to pre-ictal stage which is normal iEEG signals. The features vectors extracted from the brain signals to differentiate preictal stage from Ictal stage [9].

Figure 2 shows Seizure Predication and Classification System. The Intracranial Electroencephalography (iEEG) signals used in analysis for classification and predication of seizure are acquired from online SWEC-ETHZ iEEG database. The brain signals are acquired or recorded by using implant electrodes of type strip, grid and the depth electrodes. The iEEG signals are further preprocessed to remove power noise and extract exact band of frequency. The signals are then analyzed using wavelet domain as both time and frequency components are important. Daubechies discrete wavelet transform with eight level of decomposition is implemented on iEEG signals. The selections of sub band are done to extract delta, theta, alpha, beta and gamma frequencies. Features such as Lacunarity, Fluctuation index, Energy \& Entropy, Kolmogorov Entropy, Kurtosis and Skewness are extracted from alpha and beta sub-band of frequencies. Study shows that the seizure occurs in alpha and beta band of frequencies, so only these bands are used to extract the feature vectors [8],[12]. These features are further provided as input to classifiers to classify the iEEG signal state as ictal state which is a seizure type and non-ictal state which is of non-seizure type signal.

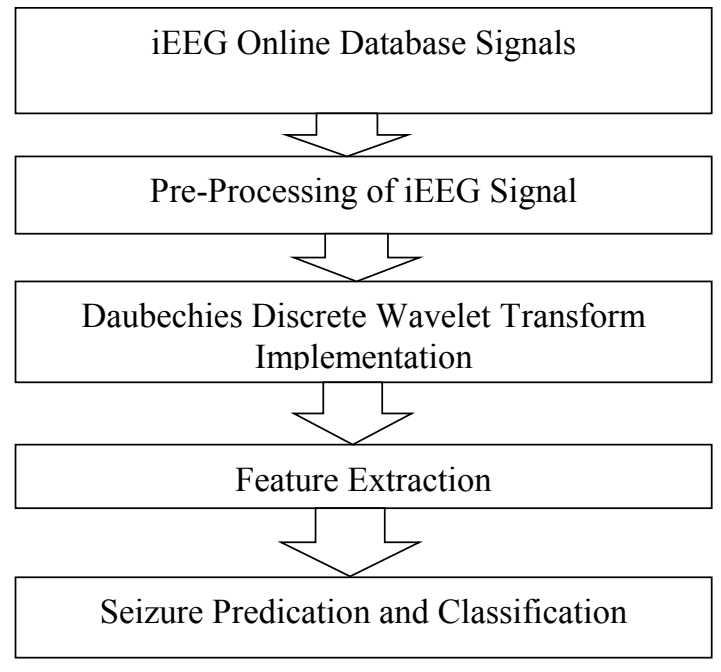

Figure 2: Seizure Predication and Classification System

\subsection{Intracranial Electroencephalography Online Database Signals}

The iEEG database is collected from SWEC-ETHZ iEEG database. The signals are recorded during pre-surgical at Sleep
Wake Epilepsy Center at university department of Neurology at the Inselspital Bern and the Integrated Systems Laboratory of the ETH Zurich. The online datasets is provided free of charge to researchers.

Their two types of dataset long term recording contain of 18 patients and short term recording consists of 16 patients. The long term database contains of 116 seizure signals of 2656 hours and short term database contains of 100 seizure signals. In our analysis for classification and predication system we have used short term recording of 100 signals of seizures records obtained from 16 patients. The signals were recorded before epilepsy surgery. The signals are acquired or recorded by using strip, grid and the depth electrodes. The signals recorded are analog in nature and are converted in digital form using 16 bit analog the digital convertors. The signals are further pre-processed by passing through fourth order Butterworth band pass filter. The lower cut-off frequency is kept as $0.5 \mathrm{~Hz}$ and upper cut-off frequency is kept as $150 \mathrm{~Hz}$ of band pass filter. The signals are then recorded on disc with the speed of $512 \mathrm{~Hz}$. Each record of signal consists of three minutes recording before the seizure which is called as preictal segment and then followed by seizure period which generally in range from $10 \mathrm{sec}$ to $1002 \mathrm{sec}$ and is called as ictal segment. After end of ictal segment or seizure again followed by three minutes of recording called as post ictal.

The signals in dataset are in zipped folders and are named with ID of patients. The seizures records are stored in .mat files, the TxM variable array is used for recording an EEG, where $\mathrm{M}$ are number of electrodes used for recording and $\mathrm{T}$ are number of sampling points [10],[11].

\subsection{Pre-Processing of iEEG Signal}

The iEEG signals during recording are added with noise, which are called as artifacts. The introduction of these artifacts affects the performance of Seizure Predication and Classification System. The iEEG signals are pre-processed by implementation of fourth order Butterworth band pass filter. The lower cut-off frequency is kept as $0.5 \mathrm{~Hz}$ and upper cutoff frequency is kept as $150 \mathrm{~Hz}$ of band pass filter. The neuronal information which is important for seizure predication and classification is from $0.5 \mathrm{~Hz}$ to $150 \mathrm{~Hz}$. This band is extracted by implementation of band pass filters. The artifacts present in iEEG are less compared to EEG signals. During recording power noise of line frequency may get introduce, which is removed by implementation of notch filter [11].

\subsection{Daubechies Discrete Wavelet Transform Implementation}

Intracranial Electroencephalography signals are non-stationary in nature. The signal needs to analyze in both 
time and frequency, so compare to traditional method discrete wavelet transform found to be more powerful and suitable method to analyze transient signals. Wavelet transform is the mathematical tool to analysis signal, which has average value as zero. Analysis of signal in wavelet domain means breaking of signal in scaling and shifting. Wavelet transform methods are used to localize the signal component better than time frequency space [14].

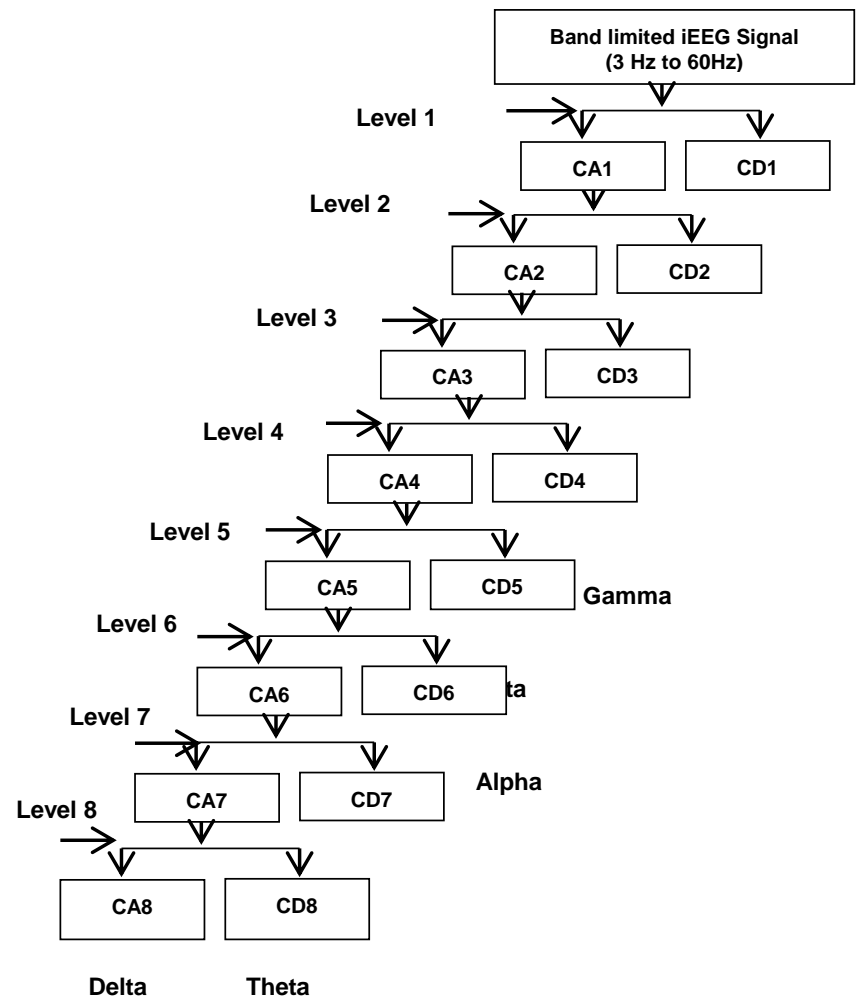

Figure 3: Daubechies Discrete Wavelet Transform Implementation Up To Eight Levels of Decomposition

Daubechies Discrete Wavelet Transform found to be most appropriate for classification and predication of epileptic seizure using iEEG database. Fig.3. represents Daubechies Discrete Wavelet Transform Implementation up to eight levels decomposition. Daubechies discrete wavelet transform with eight level of decomposition has been implemented to separate out alpha, beta, gamma, theta and delta frequency waves in iEEG signal. The alpha and beta frequency waves changes its characteristics features during ictal and non-ictal stage. The feature vector extracted from these bands gives indication about ictal and non-ictal stage.

A Discrete Wavelet Transform decomposition level gives the approximation coefficients and detail coefficients. The approximation coefficient is CA8 and detail coefficients are CD8, CD7, CD6, CD5, CD4, CD3, CD2 and CD1. The seizure normally occurs in the frequency range of $8 \mathrm{~Hz}$ to $30 \mathrm{~Hz}$, These frequency bands are termed as alpha and beta frequencies band. The detail coefficient CD7 denotes alpha band of frequency and CD6 denotes beta band of frequency. The CD6 and CD7 are used for further feature extraction [12],[17].

\subsection{Feature Extraction}

Selecting effective features is very important in Seizure Predication and Classification System. The features selected represent the characteristics of iEEG signals and must be able to differentiate between ictal stage which seizure and non-ictal stage which is non-seizure iEEG data. Feature extraction provides precise information, which helps in seizure predication. The variations in the distribution of the $\mathrm{iEEG}$ signals are measured in terms of parameters of a Gaussian process and the deviation of the distribution from Gaussian. As alpha and beta band frequencies are represented in CD7 and CD6, these detail coefficients used in feature extraction. The deviation in Gaussian of the iEEG signals can be found out by measuring the values of feature vectors such as Lacunarity, Fluctuation index, Energy \& Entropy, Kolmogorov Entropy, Lyapunov Exponent, Kurtosis and Skewness in alpha and beta band of frequencies using CD7 and CD6 detail coefficients.

Lacunarity is a scale to measure heterogeneity [15]. Low Lacunarity indicates homogeneous during pre-ictal stage and high Lacunarity indicates heterogeneous in iEEG signal during ictal stage.

Fluctuation Index is a measurement of fluctuation and intensity in iEEG signal [15],[17]. Fluctuation Index of iEEG during seizures period or ictal stage is higher than that of nonseizure periods or pre-ictal stage.

Energy and Entropy: Energy represents the strength of measured iEEG signal [6],[17]. High Energy indicates presence of ictal stage in given signal. Entropy is defined as presence of uncertainty in signal. Entropy indicates presence of event or ictal stage in measured iEEG signal [7],[13],[16].

Kolmogorov Entropy is a measurement of irregularity in iEEG signal to detect ictal stage. Kolmogorov Entropy is the entropy of source distribution generating the sequence.

Kurtosis is the measurement whether the iEEG data are peaked or flat in nature at the mean point relative to a normal distribution. The iEEG data set having high value of kurtosis is an indication that there is a peak near the mean which declines vary rapidly with heavy tails. The iEEG Data sets having low value of kurtosis is an indication that there is flat top near the mean point of the signal.

Skewness is a measurement of symmetry or lack of symmetry of iEEG data distribution. A distribution is called as symmetric if it looks exact similar to the right and left side from the Centre point. If the distribution of iEEG data set is more towards left side from the mean point than the skewness is called to be positive and vice versa. The data with symmetric distribution has a skewness value zero. 


\subsection{Seizure Predication and Classification}

The main objective of classification is to separate feature vectors in two or more classes by defining boundary between classes. Classifier is very essential part for accurate Seizure Predication and Classification System. There are number of classifiers developed over the years, out of which few classifiers such as Support Vector Machines (SVM), K-Nearest Neighbor algorithm (KNN) and Probabilistic Neural Network (PNN) were found to be accurate and widely used in classification. The above three classifiers are implemented and compared to each other to find the suitable classifier for Seizure Predication and Classification System.

Support Vector Machine (SVM) is a type of supervised machine learning algorithm which is used for iEEG classification by introducing of hyper planes in a multidimensional space which separates data of different class labels [18]. In two dimensional data as in our case of iEEG signal a single hyper plane is implemented with largest margin to separate out the data into two groups such as ictal stage and pre-ictal stage which is non ictal stage. The second classifier K-Nearest Neighbor algorithm is a clustering tool an instancebased learning methods. K-Nearest Neighbor algorithm uses by default Euclidean distance metrics function to measure similarity and minimum distance to classify the given iEEG dataset. The algorithm divides set of extracted feature vectors in $\mathrm{k}$ different clusters. The third classifier is Probabilistic Neural Network (PNN) which is a supervised neural network implemented for classification of iEEG data. PNN is a type of Artificial Neural Network which has four layers named as input layer, pattern layer, summation layer and output layer. PNN has disadvantage as it requires more memory space but are faster and generates accurate prediction [12].

\section{Performance Evaluation}

The performance evaluation of Seizure Predication and Classification System is carried out by using iEEG signal acquired from online dataset. The short term dataset of 16 patients are named in folder from ID1 to ID16. There are total collections of 100 seizure signals from 16 patients used in analysis. The iEEG signal dataset used in experiment are collected using strip, grid and depth electrodes. Each iEEG signal recorded consists of three minutes of recording before occurrence of seizure which is called as pre-ictal segment. The pre-ictal segment is followed by the seizure period which is called as ictal segment. The ictal segment duration is different for different patient and ranges from 10 seconds to 1002 seconds. The ictal segment is followed by three minutes recording called as post ictal segment. Every iEEG signal record has three minutes of pre-ictal segment then ictal segment depends on patient and final three minutes of postictal segment [10],[11]. The analysis of pre-ictal segment and ictal segment is done to classify as seizure and non-seizure. The analysis of pre-ictal segment is done to predicate the seizure. The feature vector helps in marking ictal stage and non-ictal stage and to predicate seizure.

\subsection{Performance Evaluation of iEEG Signal for Classification}

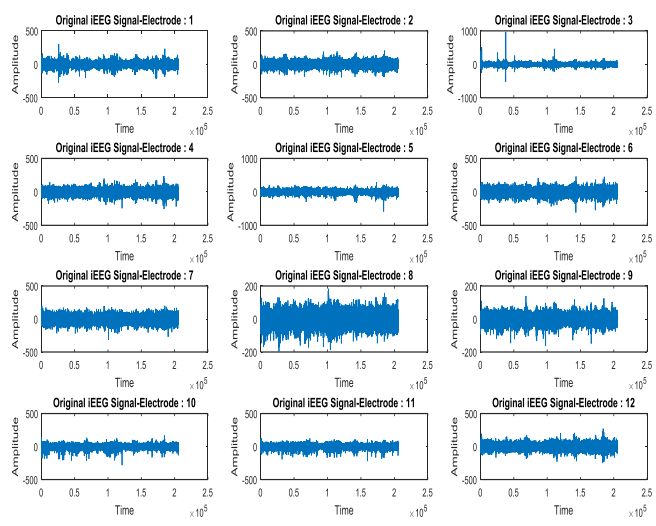

Figure 4: iEEG Signal Acquired from Strip and Grid Electrodes

Figure 4 represents plotting of iEEG electrode of ID1 patient acquired using 47 strip and Grid electrodes. Out of 47 Electrodes 12 electrodes signal is plotted in Figure 4.

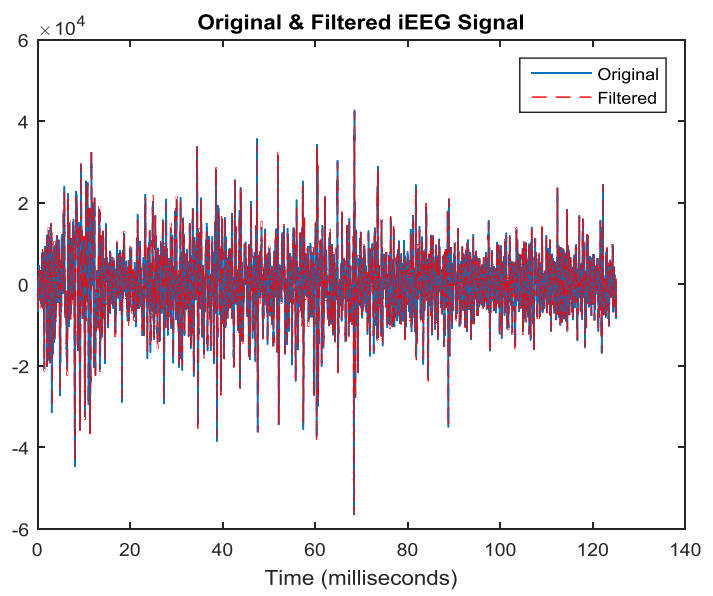

Figure 5: iEEG Signal Before and after Pre-Processing

Figure 5 shows the original and filtered iEEG signal. The original signal is plotted before pre-processing and the filtered signal is plotted after pre-processing. The iEEG signal demonstrate here is of patient ID1 with 47 electrodes which is pre-processed by passing through notch filter to remove power noise. The signal is than is further passed through band pass 
filter of frequency $3 \mathrm{~Hz}$ to $60 \mathrm{~Hz}$ to extract the exact band of frequency, where the seizure generally occurs.

Daubechies Discrete Wavelet Transform up to eight levels of decomposition is implemented and the feature vectors are found out. The Feature vectors are classified by implementation of three classifiers to classify the iEEG signal as Pre-ictal segment and Ictal segment. The classification of iEEG signal is carried out electrode wise. After extracting or separating Pre-ictal segment and Ictal segments from total record these segments are pre-processed electrode wise. Discrete wavelet decomposition is implemented and feature extraction of segments. A testing set of 50 seizure iEEG signals is analysed with 3037 total electrodes. From the electrodes 3037 Pre-Ictal segment and 3037 Ictal segments were tested by seizure detection system. The classification of iEEG signals is done as Pre-ictal segment and Ictal segment.

\subsection{Performance Evaluation of iEEG signal for Seizure Predication}

Seizures are the sudden change in brain electrical voltage. There was no warning received before start of seizure (ictal stage) by person suffering from Epilepsy in past. Predication of seizure helps patients to acquire advance warning and may prevent seizure by anti-seizure drugs. The predication of seizure is done by analysis of Pre-Ictal segment of three Minutes, which is separated out or extracted from the total iEEG record. Analysis of Pre-Ictal segment is done electrode wise by pre-processing the signal to remove power noise and extract required band of frequencies. Discrete Wavelet Transform with eight level of Decomposition is implemented and feature vectors are extracted from alpha band and beta band of frequencies. The features of Pre-Ictal segment and Features found out of training set signals are compared to differentiate the Pre-ictal stage and start of Ictal stages. Various feature vectors such as Lacunarity, Kolmogorov Entropy, Entropy, Energy, Kurtosis and Skewness are found out and analysed to differentiate between Pre-Ictal and start Ictal stage. The exact timing is found and fix when the Pre-Ictal segment changes to Ictal segment with respect to change in features.

\section{Result of Seizure Classification and Predication}

The proposed Seizure Predication and Classification System performance is measured in terms of statistical parameters such as Sensitivity, Specificity, Accuracy and True Predictive Value. The Sensitivity, Specificity, Accuracy and True Predictive Value of the above three classifiers are found out and compared to find the best suited classifier for Seizure Predication and Classification System for iEEG signals.
Sensitivity: It is measurement of number of true positives Ictal segment to the total number of Ictal segment labeled by the iEEG experts. True positive indicates detected Ictal segment by our algorithm was also identified as Ictal segment by the iEEG experts.

$$
\text { Sensitivity }=\mathrm{TP} /(\mathrm{TP}+\mathrm{FN})
$$

Specificity: It is measurement of number of true Pre-ictal segment to the total number of pre-ictal segment labeled by the iEEG experts. True negative indicates a signal labeled as Preictal segment both by our algorithm as well as by the iEEG experts.

$$
\text { Specificity }=\mathrm{TN} /(\mathrm{TN}+\mathrm{FP})
$$

Accuracy: It is measurement of number of correctly identified Ictal segment and Pre-ictal segment signals to the total number of signals segment.

$$
\text { Accuracy }=(\mathrm{TN}+\mathrm{TP}) /(\mathrm{TN}+\mathrm{TP}+\mathrm{FN}+\mathrm{FP})
$$

True Predicative Value: It is measurement of number of Ictal segment identified by our algorithm as well as labeled by iEEG experts.

$$
\text { True Predictive Value }=\mathrm{TP} /(\mathrm{TP}+\mathrm{FP})
$$

Where True Positive (TP) is number of Ictal segment identified by our algorithm and also marked by expert. False Negative (FN) is number of Ictal segments missed by our algorithm but are been identified and marked by expert. True Negative (TN) is number of Pre-ictal segment identified by our algorithm and also marked by expert and finally False Positive (FP) is defined as pre-ictal segment identified as Ictal segment by our algorithm.

\subsection{Result of iEEG Classification}

A training set is formed of 50 seizure iEEG signals which consist of 3113 electrodes. Pre-Ictal segment of 3113 and Ictal segments of 3113 were extracted. A testing set of 50 seizure iEEG signals were analyzed having 3037 electrodes. Pre-Ictal segment of 3037 and Ictal segment of 3037 were tested with implementation of three classifiers.

Testing Set= Pre-ictal segment 3037 and Ictal segment 3037 with total 6074 segments

Training Set= Pre-ictal segment 3113 and Ictal segment 3113 with total 6226 segments

Table 1: Statistical Parameter Comparison of Classifiers

\begin{tabular}{|c|c|c|c|c|}
\hline Classifier & $\begin{array}{c}\text { Sensitivity } \\
\mathbf{\%}\end{array}$ & $\begin{array}{c}\text { Specificity } \\
\mathbf{\%}\end{array}$ & $\begin{array}{c}\text { Accuracy } \\
\mathbf{\%}\end{array}$ & $\begin{array}{c}\text { TPV } \\
\mathbf{\%}\end{array}$ \\
\hline SVM & 77.84 & 100 & 88.92 & 100 \\
\hline KNN & 100 & 97.37 & 98.68 & 97.43 \\
\hline PNN & 83.04 & 100 & 91.52 & 100 \\
\hline
\end{tabular}


The Table 1 shows the comparison between Support Vector Machines, K-Nearest Neighbor algorithm and Probabilistic Neural Network classifiers used in our system for same iEEG dataset in terms of statistical parameters such as Sensitivity, Specificity, Accuracy and True Predicative Value.

\subsection{Result of Seizure Predication}

Table 2 shows the example of ID1 patient whose seizure recording file number seven (1Sz7.mat) is electrode wise analyzed for predication of seizure. The ID1 Patient has 47 grid electrodes implanted through which the signals are recoded.

Table 2: Predication of Ictal Stage

\begin{tabular}{|c|c|c|}
\hline \multicolumn{3}{|c|}{ Patient File Name: 1Sz7.mat } \\
\hline $\begin{array}{l}\text { Electrode } \\
\text { Number }\end{array}$ & $\begin{array}{l}\text { Predication } \\
\text { of Ictal in } \\
\text { Seconds }\end{array}$ & $\begin{array}{l}\text { Predication of } \\
\text { Ictal in Minutes }\end{array}$ \\
\hline 1 & $164 \mathrm{sec}$ & $2.73 \mathrm{~min}$ \\
\hline 2 & $172 \mathrm{sec}$ & $2.87 \mathrm{~min}$ \\
\hline 3 & $172 \mathrm{sec}$ & $2.87 \mathrm{~min}$ \\
\hline 4 & $156 \mathrm{sec}$ & $2.60 \mathrm{~min}$ \\
\hline 5 & $172 \mathrm{sec}$ & $2.87 \mathrm{~min}$ \\
\hline 6 & $164 \mathrm{sec}$ & $2.73 \mathrm{~min}$ \\
\hline 7 & $109 \mathrm{sec}$ & $1.82 \mathrm{~min}$ \\
\hline 8 & $172 \mathrm{sec}$ & $2.87 \mathrm{~min}$ \\
\hline 9 & $164 \mathrm{sec}$ & $2.73 \mathrm{~min}$ \\
\hline 10 & $164 \mathrm{sec}$ & $2.73 \mathrm{~min}$ \\
\hline 11 & $164 \mathrm{sec}$ & $2.73 \mathrm{~min}$ \\
\hline 12 & $156 \mathrm{sec}$ & $2.60 \mathrm{~min}$ \\
\hline 13 & $172 \mathrm{sec}$ & $2.87 \mathrm{~min}$ \\
\hline 14 & $172 \mathrm{sec}$ & $2.87 \mathrm{~min}$ \\
\hline 15 & $164 \mathrm{sec}$ & $2.73 \mathrm{~min}$ \\
\hline 16 & $148 \mathrm{sec}$ & $2.47 \mathrm{~min}$ \\
\hline 17 & $164 \mathrm{sec}$ & $2.73 \mathrm{~min}$ \\
\hline 18 & $172 \mathrm{sec}$ & $2.87 \mathrm{~min}$ \\
\hline 19 & $31 \mathrm{sec}$ & $0.52 \mathrm{~min}$ \\
\hline 20 & $172 \mathrm{sec}$ & $2.87 \mathrm{~min}$ \\
\hline 21 & $172 \mathrm{sec}$ & $2.87 \mathrm{~min}$ \\
\hline 22 & $164 \mathrm{sec}$ & $2.73 \mathrm{~min}$ \\
\hline 23 & $156 \mathrm{sec}$ & $2.60 \mathrm{~min}$ \\
\hline 24 & $172 \mathrm{sec}$ & $2.87 \mathrm{~min}$ \\
\hline 25 & $172 \mathrm{sec}$ & $2.87 \mathrm{~min}$ \\
\hline 26 & $172 \mathrm{sec}$ & $2.87 \mathrm{~min}$ \\
\hline 27 & $172 \mathrm{sec}$ & $2.87 \mathrm{~min}$ \\
\hline 28 & $164 \mathrm{sec}$ & $2.73 \mathrm{~min}$ \\
\hline 29 & $164 \mathrm{sec}$ & $2.73 \mathrm{~min}$ \\
\hline 30 & $172 \mathrm{sec}$ & $2.87 \mathrm{~min}$ \\
\hline 31 & $164 \mathrm{sec}$ & $2.73 \mathrm{~min}$ \\
\hline 32 & $172 \mathrm{sec}$ & $2.87 \mathrm{~min}$ \\
\hline
\end{tabular}

\begin{tabular}{|l|l|l|}
\hline 33 & $164 \mathrm{sec}$ & $2.73 \mathrm{~min}$ \\
\hline 34 & $172 \mathrm{sec}$ & $2.87 \mathrm{~min}$ \\
\hline 35 & $172 \mathrm{sec}$ & $2.87 \mathrm{~min}$ \\
\hline 36 & $140 \mathrm{sec}$ & $2.33 \mathrm{~min}$ \\
\hline 37 & $140 \mathrm{sec}$ & $2.33 \mathrm{~min}$ \\
\hline 38 & $164 \mathrm{sec}$ & $2.73 \mathrm{~min}$ \\
\hline 39 & $172 \mathrm{sec}$ & $2.87 \mathrm{~min}$ \\
\hline 40 & $8 \mathrm{sec}$ & $0.13 \mathrm{~min}$ \\
\hline 41 & $172 \mathrm{sec}$ & $2.87 \mathrm{~min}$ \\
\hline 42 & $148 \mathrm{sec}$ & $2.47 \mathrm{~min}$ \\
\hline 43 & $140 \mathrm{sec}$ & $2.33 \mathrm{~min}$ \\
\hline 44 & $148 \mathrm{sec}$ & $2.47 \mathrm{~min}$ \\
\hline 45 & $164 \mathrm{sec}$ & $2.73 \mathrm{~min}$ \\
\hline 46 & $148 \mathrm{sec}$ & $2.47 \mathrm{~min}$ \\
\hline 47 & $148 \mathrm{sec}$ & $2.47 \mathrm{~min}$ \\
\hline
\end{tabular}

Table 2 shows electrode wise predication of Ictal (seizure) period starting in terms seconds and Minutes. The seizure is predicated for most of electrodes before maximum period of 172 seconds (2.87 minutes) and minimum period on 8 seconds (0.13 minutes) which is on Electrode number 40.

\section{Conclusion}

Seizure Predication and Classification System is important tool to analysis and predicate epileptic seizure in iEEG signals. In this paper Predication and Classification System was proposed and iEEG signal was analyzed in wavelet transform using Daubechies Discrete Wavelet Transform by eight level of decomposition. Statistical features such as Lacunarity, Fluctuation Index, Energy \& Entropy, Kolmogorov Entropy, Kurtosis and Skewness were extracted from alpha band and beta band of frequencies. Classifiers Support Vector Machines, K-Nearest Neighbor algorithm and Probabilistic Neural Network were implemented to classify as ictal segment and non-ictal segment. Performance analysis of classifier was carried out in terms of Sensitivity, Specificity, Accuracy and True Predictive Value. The Experimental results of classification of online standard iEEG datasets shows that Seizure Predication and Classification System achieves a highest Accuracy of $98.68 \%$,Specificity of $97.37 \%$,Sensitivity of $100 \%$ and True Predicative Value of $97.43 \%$ for K-Nearest Neighbor algorithm. Daubechies Discrete Wavelet Transform with K-Nearest Neighbor classifier algorithm founds to better for classification of iEEG signal. Seizure predication is done electrode wise maximum before 172 seconds or minimum before 8 seconds by analysis of pre-ictal segment. Seizure predication and further control of seizure with anti-epileptic drug helps in improving quality of life in Epilepsy patients. 


\section{Acknowledgment}

Authors would like to thank Sleep-Wake-Epilepsy-Center (SWEC) of the University Department of Neurology at the Inselspital Bern and the Integrated Systems Laboratory of the ETH Zurich for providing iEEG online dataset free of charge for research work.

\section{References}

[1] Saeid Sanei and J.A. Chambers, "EEG Signal Processing," In Centre of Digital Signal Processing, Cardiff University, UK, John Wiley \& Sons Ltd, 2007.

[2] Yinxia Liu,Weidong Zhou, Qi Yuan and Shuangshuang Chen, "Automatic Seizure Detection Using Wavelet Transform and SVM in Long-Term Intracranial EEG," IEEE Transactions on Neural Systems and Rehabilitation Engineering, vol.20, no.6, pp. 749-755, 2012.

[3] Gardner, A. B., Krieger, A. M., Vachtsevanos, G., \& Litt, B, "One-class Novelty Detection for Seizure Analysis from Intracranial EEG," Journal of Machine Learning Research, vol.7, no.6, pp. 1025-1044, 2006.

[4] Zhou, W., Liu, Y., Yuan, Q., \& Li, X., "Epileptic Seizure Detection Using Lacunarity and Bayesian Linear Discriminant Analysis in Intracranial EEG," IEEE Transactions on Biomedical Engineering, vol.60, no.12, pp. 3375-3381, 2013.

[5] Pawar, S., Chougule, S. R., \& Tirmare, A. H., “ Diagnosis of Epilepsy a Neurological Disorder Using Electroencephalogram (EEG)," International Journal of Modern Trends in Engineering and Research, vol.4, no.6, pp. 144-149, 2017.

[6] Sanjay S. Pawar, S.R.Chougule, "Diagnosis and Analysis of Epileptic Seizure Neurological Disorder Using Electroencephalography," IOSR Journal of VLSI and Signal Processing (IOSR-JVSP), vol.9, no.1, pp. 23-27, 2019.

[7] S. S. Pawar and S. R. Chougule, "Diagnosis and Classification of Epileptic Seizure a Neurological Disorder Using Electroencephalography," In: 2nd International Conference on Intelligent Computing, Instrumentation and Control Technologies (ICICICT), Kannur, Kerala, India. pp. 159-163, 2019. doi: 10.1109/ICICICT46008.2019.8993378.

[8] S. S. Pawar and S. R. Chougule, "Diagnosis of Epileptic Seizure a Neurological Disorder by Implementation of Discrete Wavelet Transform Using Electroencephalography," International Conference on Communication Systems and Networks (ComNet 2019), Trivandrum, Kerala, India, 2019.

[9] Zisheng Zhang, Keshab K. Parhi, "Low-Complexity Seizure Predication From iEEG/sEEG Using Spectral Power and Ratios of Spectral Power," IEEE Transactions of Biomedical circuits and Systems, vol.10, no.3, pp. 693-706, 2016.

[10] Alessio Burrello, Kaspar Schindler, Luca Benini, Abbas Rahimi, "One-shot Learning for iEEG Seizure Detection Using End-to-end Binary Operations: Local Binary
Patterns with Hyperdimensional Computing," In proceedings of the IEEE Biomedical Circuits and Systems Conference (BioCAS), Cleveland, OH, pp.1-4, 2018.

[11] Alessio Burrello, Kaspar Schindler, Luca Benini, Abbas Rahimi, "Hyperdimensional Computing with Local Binary Patterns: One-shot Learning of Seizure Onset and Identification of Ictogenic Brain Regions using Short-time iEEG Recordings," IEEE Transactions on Biomedical Engineering (TBME), vol.67, no.2, pp.601-613, 2019.

[12] Pawar S.S., Chougule S.R., "Diagnosis of Epileptic Seizure a Neurological Disorder by Implementation of Discrete Wavelet Transform Using Electroencephalography," In: Jayakumari J., Karagiannidis G., Ma M., Hossain S. (eds) Advances in Communication Systems and Networks. Lecture Notes in Electrical Engineering, vol.656.Springer,Singapore. https://doi.org/10.1007/978-981-15-3992-3 59, 2020.

[13] Pawar, S. S., \& Narayankar, K. G. , "Tsallis Entropy Based Seizure Detection," International Journal of Innovations in Engineering Research and Technology," vol.4, no.6, pp. 86-92, 2017.

[14]Pawar, S. S., \& Suryawanshi, M. N., "Epilepsy Seizure Detection Using Wavelet Based By Artifact Reduction," International Journal of Innovations in Engineering Research and Technology, vol.4, no.6, pp.11-18, 2017.

[15] Weidong Zhou, Yinxia Liu, Qi Yuan, and Xueli Li, "Epileptic Seizure Detection Using Lacunarity and Bayesian Linear Discriminant Analysis in Intracranial EEG," IEEE Transactions on Biomedical Engineering, vol.60, no.12, pp. 3375-3381, 2013.

[16] Harlalka, V., Puntambekar, V. P., Raviteja, K., \& Mahalakshmi, P., "Detection of Epileptic Seizure Using Wavelet Analysis based Shannon Entropy, Logarithmic Energy Entropy and Support Vector Machine," International Journal of Engineering \& Technology, vol.7, no. 4.10, pp.: 935-939, 2018.

[17] Yinxia Liu, Weidong Zhou, Qi Yuan, and Shuangshuang Chen, "Automatic Seizure Detection Using Wavelet Transform and SVM in Long-Term Intracranial EEG," IEEE Transactions on Neural Systems and Rehabilitation Engineering, vol.20, no.6, pp.749-755, 2012.

[18] Bhuvaneswari, P., \& Kumar, J. S., "Support Vector Machine Technique for EEG Signals," International Journal of Computer Applications, vol.63, no.13, pp.1-5, 2013.

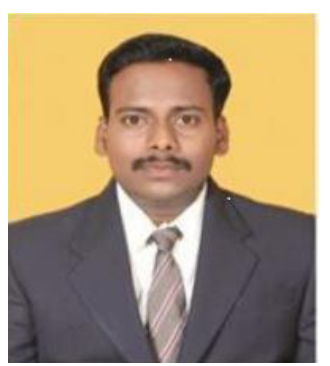

Sanjay S. Pawar is born in Miraj, Maharashtra (India) on 7th July 1980. $\mathrm{He}$ is graduate in Electronics Engineering from Walchand College of Engineering, Sangli, Maharashtra (India) in 2003 and has done his post graduate in Electronics and Telecommunication Engineering from Kolhapur Institute of Technology, Kolhapur, Maharashtra (India). 
$\mathrm{He}$ is presently working as Assistant Professor in Department of Electronics and Telecommunication Engineering at Bharati Vidyapeeth's College of Engineering, Kolhapur, Maharashtra (India). He has 15 years of teaching experience and 1 year of industrial experience. He has published more than 40 research papers in journals and conference proceedings. His specialization includes Embedded System, Signal processing.

Mr Pawar is member of IAENG International Association of Engineers.

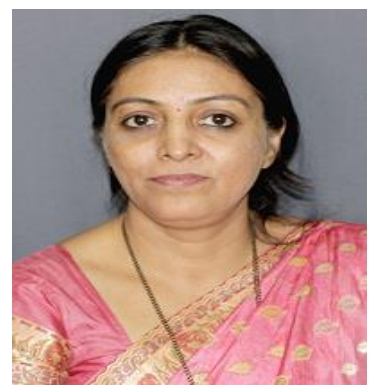

Dr. Sangeeta R. Chougule has acquired Ph.D degree in Electronics Engineering and presently working as professor in Electronics \& Telecommunication Engineering at Kolhapur Institute of Technology, Kolhapur, Maharashtra (India).

She has 26 years of teaching, 2 years of industrial and 6 years of research experience. She has presented \& published 77 research papers in National /International Journal/Conference. Her specialization includes Digital signal processing and Image processing.

Dr. Chougule is Chairman IETE and member of ISTE, IJERIA and IJMSEA.

\section{Author Contributions}

Sanjay S. Pawar carried out the data collection, implementation of Algorithm, simulation and execution of experiment in Matlab.

Sangeeta R. Chougule has guided for data collection, implementation of Algorithm, simulation and execution of experiment in Matlab.

\section{Sources of funding for Research}

There has been no financial support for this work that could influence its outcomes.

\section{Creative Commons Attribution License 4.0 (Attribution 4.0 International, CC BY 4.0)}

This article is published under the terms of the Creative Commons Attribution License 4.0

https://creativecommons.org/licenses/by/4.0/deed.en_US 